\title{
The Use of Quantum-Chemical Semiempirical Methods to Calculate the Lattice Energies of Organic Molecular Crystals. Part II: The Lattice Energies of $\alpha$ - and $\beta$-Oxalic Acid $(\mathrm{COOH})_{2}$
}

\author{
Gerhard Raabe \\ Institut für Organische Chemie der Rheinisch-Westfälischen Technischen Hochschule Aachen, \\ Prof.-Pirlet-Straße 1, D-52056 Aachen \\ Reprint requests to Priv.-Doz. Dr. G. R.; Fax: +49 (0)241 8092385 , \\ Email: Gerd.Raabe@thc.rwth-aachen.de \\ Z. Naturforsch. 57 a, 961-966 (2002); received August 9, 2002 \\ Dedicated to Prof. Dr. Welf Bronger on the occasion of his 70th birthday \\ Presented in part at the 9. Jahrestagung der Deutschen Gesellschaft für Kristallographie, March \\ 12 - 15. 2001, Bayreuth, Germany and at the XIX Congress and Assembly of the International \\ Union of Crystallography, August 6 - 15. 2002, Geneva, Switzerland.
}

\begin{abstract}
The lattice energies $\left(\Delta E_{\text {lat }}\right)$ of $\alpha$ - and $\beta$-oxalic acid $\left((\mathrm{COOH})_{2}\right)$ have been calculated using a recently introduced semiempirical quantum-chemical procedure. Within the framework of this method the lattice energy $\left(\Delta E_{\text {lat }}\right)$ is evaluated as the sum of the semiempirically calculated intermolecular dispersion $\left(\Delta E_{\text {dis }}\right)$, induction $\left(\Delta E_{\text {ind }}\right)$, repulsion $\left(\Delta E_{\text {rep }}\right)$, and electrostatic energy $\left(\Delta E_{\mathrm{els}}\right)$. The lattice energies of the two polymorphs of oxalic acid obtained in this way correlate not only with the results of other calculations but also with the experimentally determined heats of sublimation in that the $\alpha$-modification, which has a somewhat higher heat of sublimation, is slightly more stable than the $\beta$-polymorph. However, additional quantum-chemical calculations at the non-empirical $a b$ initio level (e. g. ZPE+MP2(FC)/6-311++G**//MP2(FC)/6-311++G**) revealed that the absolute values of the lattice energies and the heats of sublimation are not directly comparable to each other because the structures of the $(\mathrm{COOH})_{2}$ molecules in the crystal lattices of both polymorphs differ significantly from that of the most stable form of the free molecule in the gasphase. At about $4.3 \mathrm{kcal} / \mathrm{mol}$ the calculated energy difference between the structure of the molecule in the solid state and the energetically most favourable conformation of the free $(\mathrm{COOH})_{2}$ molecule in the gasphase is much smaller than that in the recently described case of $\alpha$-glycine $(28 \pm 2 \mathrm{kcal} / \mathrm{mol})$. However, even such a small difference might be the source of serious problems if the heats of sublimation are employed to fit parameters to be used in the optimization of crystal packings.
\end{abstract}

Key words: Lattice Energies; Oxalic Acid; Calculations; Semiempirical Methods.

\section{Introduction}

The solid state structures of the two polymorphs of oxalic acid have been described by several authors [1 - 4].

(i) The $\alpha$-polymorph crystallizes in the orthorhombic space group Pcab (No. 61) with four centrosymmetric molecules in a unit cell with the lattice constants $a=6.548(5), b=7.844(5)$, and $c=$ $6.086(5) \AA[3]$. Each of the molecules is hydrogenbonded to four neighbours $(\mathrm{O} 1-\mathrm{H}$... $\mathrm{O} 2$ : 1.886(1), $\mathrm{O} 1 \cdots \mathrm{O} 2: 2.702(1) \AA$ ) resulting in folded layers extending approximately parallel to the $b c$ plane. At 2.879(2) $\AA$ the shortest distance between these layers is surprisingly small $(\mathrm{O} 2 \ldots \mathrm{C})$ and significantly below the sum of the isotropic van der Waals radii of carbon and oxygen (1.70 and $1.52 \AA$ ).

(ii) The $\beta$-polymorph crystallizes in the monoclinic space group $P 2{ }_{1} / c$ (No. 14) with two centrosymmetric molecules in a unit cell with the parameters $a=$ 5.330(4), $b=6.015(5), c=5.436(4) \AA$, and $\beta=$ $115.83(3)^{\circ}[3]$. Each molecule is bonded to two neighbours $(\mathrm{O} 1-\mathrm{H} \ldots \mathrm{O} 2: 1.727(23), \mathrm{O} 1 \ldots \mathrm{O} 2: 2.674(3) \AA)$, resulting in chains extending parallel to the $a$ axis. In 
this case the shortest distance of 2.900(2) $\AA$ occurs between the carbonyl oxygen and carbon of neighbouring chains.

The experimental heats of sublimation $\left(\Delta H_{\text {sub }}\right)$ and the derived lattice energies $\left(\Delta E_{\text {lat }}\right)$ of the two polymorphs are quite similar. Thus, from their vapour pressure measurements Bradley and Cotson [5, 6] derived heats of sublimation of 23.4 for the $\alpha$ - and of $22.3 \mathrm{kcal} / \mathrm{mol}$ for the $\beta$-polymorph. Using the approximate relationship $\Delta E_{\text {lat }}=-\Delta H_{\text {sub }}-2 R T$, these heats of sublimation correspond to experimental lattice energies of -24.6 and $-23.5 \mathrm{kcal} / \mathrm{mol}$ at $298 \mathrm{~K}$. Starting from de Kruif's unpublished data, Derissen and Smit [7] obtained experimental lattice energies of -23.4 and $-23.1 \mathrm{kcal} / \mathrm{mol}$ for the $\alpha$ - and the $\beta$-modification and, therefore, even a smaller relative stability of the two forms. In a more recent paper de Wit et al. [8] reported experimental heats of sublimation of 23.5 and $22.1 \mathrm{kcal} / \mathrm{mol}$ for the $\alpha$ - and the $\beta$-form. These values agree well with the earlier results by Bradley and Cotson [5].

Calculations on the packing and the lattice energies of the two polymorphs of oxalic acid have been performed by several authors [9 - 17] employing empirical as well as non-empirical intermolecular potentials. Using the MCMS force field ${ }^{\text {a) }}$ to optimize the crystal energy, Momany et al. obtained $-25.9 \mathrm{kcal} / \mathrm{mol}$ for the packing energy of $\alpha$-oxalic acid [9]. Employing their newly derived empirical interatomic potential functions, Derissen and Smit [7] calculated a lattice energy of $-23.5 \mathrm{kcal} / \mathrm{mol}$ for the same modification of the experimental structure, while a value of $-24.9 \mathrm{kcal} / \mathrm{mol}$ was obtained for the optimized lattice. Testing different parameter sets for the $\beta$-polymorph the same authors obtained lattice energies of about $-23.0 \mathrm{kcal} / \mathrm{mol}$ for the experimental and between -24.1 and $-25.1 \mathrm{kcal} / \mathrm{mol}$ for the optimized crystal structures. Hagler et al. compared the performances of three different force fields in crystal structure optimizations of some carboxylic acids $[14,15]$. With a 9-6-1 CFF and a 12-6-1 potential ${ }^{\text {a) }}$ they obtained slightly higher stabilities for the $\alpha$-polymorph (9-6-1: $\alpha=-27.4, \beta=-26.9 \mathrm{kcal} / \mathrm{mol} .12-6-1: \alpha=$ $-29.0, \beta=-28.6 \mathrm{kcal} / \mathrm{mol}$ ), while the MCMS force

\footnotetext{
a) The MCMS force field is described in detail in $[9,10] .9-6-1$ is a potential where the repulsive, the van der Waals, and the electrostatic terms are represented by $r^{-9}, r^{-6}$, and $r^{-1}$ functions, respectively. 12-6-1 differs from the 9-6-1 potential in that the repulsive part is represented by a $r^{-12}$ term. 6-exp employs the usual $r^{-6}$ van der Waals term and an exponential repulsion function.
}

field yielded a somewhat lower energy for the $\beta$-form ( $\alpha=-27.5, \beta=-28.7 \mathrm{kcal} / \mathrm{mol}$ ) [14]. Using experimental crystal structures, dipole moments calculated for the molecular structures in the crystal lattice, and three different 9-6-1 potentials, Lifson et al. [13] obtained lattice energies which are equal for both polymorphs or slightly more negative for the $\alpha-(-24.7$ to $-24.9 \mathrm{kcal} / \mathrm{mol})$ than for the $\beta$-modification $(-24.6$ to $-24.7 \mathrm{kcal} / \mathrm{mol})$. In contrast, three different $12-6-1$ force fields resulted in consistently more negative lattice energies for the $\beta$ - $(-24.2$ to $-24.4 \mathrm{kcal} / \mathrm{mol})$ than for the $\alpha$-polymorph $(-23.3$ to $-23.5 \mathrm{kcal} / \mathrm{mol})$ [13]. Excluding explicit electrostatic terms and using an empirical 6-exp potential, Gavezzotti and Filippini [12a] obtained a significantly higher stability for the $\beta$-polymorph $(\alpha=-17.6, \beta=-21.3 \mathrm{kcal} / \mathrm{mol})$. In a more recent paper [12b] Gavezzotti calculated the electrostatic and the induction energy for the $\alpha$-polymorph by numerical integration of electron densities calculated at the MP2/6-31G** level (vide infra).

Non-empirical atom-atom potentials have been used by Smit et al. [11] to study the intermolecular interactions in crystals of carboxylic acids. Using these potentials in lattice structure optimizations with a steepest-descent method they obtained a significantly higher stability for the $\beta-(-31.8 \mathrm{kcal} / \mathrm{mol})$ than for the $\alpha$-form $(-25.9 \mathrm{kcal} / \mathrm{mol})$. Use of experimental lattice structures with the same potentials restored the observed order of stability, i. e. lattice energies of -25.1 and $-23.1 \mathrm{kcal} / \mathrm{mol}$ have been obtained for the $\alpha$ - and $\beta$-polymorph. Employing the empirical potentials derived in [7] the same authors calculated almost equal lattice energies for the two modifications ( $\alpha=$ $-24.5, \beta=-24.1 \mathrm{kcal} / \mathrm{mol}$ ) [11].

Using several non-empirical intermolecular potentials, Price and Nobeli [16] calculated lattice energies which cover the range between -22.2 and $-27.9 \mathrm{kcal} / \mathrm{mol}$ for the $\alpha-$ and -22.4 and $-31.5 \mathrm{kcal} / \mathrm{mol}$ for the $\beta$-form. They obtained energy differences between 0.7 and $1.7 \mathrm{kcal} / \mathrm{mol}$ in favour of the $\beta$-polymorph for those potentials which reproduce both crystal structures.

Combining an $a b$ initio-based electrostatic model with an empirical atom-atom potential and experimental crystal structures, Beyer and Price [17] obtained values of -23.9 for the $\alpha$ - and $-26.6 \mathrm{kcal} / \mathrm{mol}$ for the $\beta$-polymorph, which slightly changed to -24.2 and $-27.4 \mathrm{kcal} / \mathrm{mol}$ upon optimization of the crystal lattices. 
The results of most calculations agree with the experimental finding that the energy difference between the two polymorphs is quite small. However, they partly contradict each other as far as the relative stability of the two forms is concerned. Such a small energy difference provides an excellent test for any method to calculate lattice energies. It is, for example, of interest to see how a recently introduced semiempirical method, which worked reasonably well in the case of the three polymorphs of glycine [18], works in this case.

\section{Computational Method}

In this paper the lattice energy $\left(\Delta E_{\text {lat }}\right)$ of a molecular crystal is defined as the change of energy associated with a hypothetical process in which one mole of its initially infinitely separated (i.e. non-interacting) constituents ${ }^{\mathrm{b})}$ combines to form a crystal lattice. As described in [18] the lattice energy $\left(\Delta E_{\text {lat }}\right)$ in the corresponding crystal lattice has been calculated on an atom-in-molecule level, where $\Delta E_{\text {lat }}$ is approximated by the sum of the dispersion $\left(\Delta E_{\mathrm{dis}}\right)$, electrostatic $\left(\Delta E_{\text {els }}\right)$, induction $\left(\Delta E_{\text {ind }}\right)$, and (closed shell- or Pauli-) repulsion energy $\left(\Delta E_{\text {rep }}\right)$ :

$$
\Delta E_{\text {lat }}=\Delta E_{\text {dis }}+\Delta E_{\text {els }}+\Delta E_{\text {ind }}+\Delta E_{\text {rep }} .
$$

A detailed desciption of the calculation of the single components is given in [18]. It is, therefore, sufficient to concentrate on the differences between the method described previously and the procedure employed in this paper. These differences mainly concern the induction energy. In [18] atom-in-molecule polarizability tensors, calculated within the framework of the MINDO/3 [19] method as described in [20], have been used to calculate the induction energy. Since such polarizability tensors have not been available for all the semiempirical methods used in this paper, we employed scalar polarizabilities $\alpha_{a}\left(\alpha_{\mathrm{H}}=0.426\right.$, $\alpha_{\mathrm{C}}=1.594, \alpha_{-\mathrm{O}-}=0.749, \alpha_{=\mathrm{O}}=0.682 \mathrm{~A}^{3}$ ) which can be obtained from the data in $[20]^{\mathrm{c})}$, and used the expression

$$
\Delta E_{\text {ind }}=-\frac{1}{2} \sum_{a} \alpha_{a}\left|\boldsymbol{E}_{a}\right|^{2}
$$

\footnotetext{
b)i.e. the molecules in a structure as they occur in the crystal lattice.

c) To obtain these values, insert the increments listed in Table 2 of [20] into the correlation equation given in the heading of Table 3 of the same paper.
}

to calculate the induction energy. Here $\boldsymbol{E}_{a}$ is the total electric field caused by the charges of all atoms not belonging to the reference molecule at the position of atom $a$. The same atomic polarizabilities have been used in the calculation of the dispersion energy utilizing the London formula.

The atomic charges entering the calculation of the electrostatic and induction energy, the ionization potentials (Koopmans) to be used in the calculation of the dispersion energy, and the molecular orbitals employed to calculate the repulsion energy have been calculated with the corresponding semiempirical methods (MINDO/3 [19], MNDO [21], CNDO/2 [22], INDO [23], AM1 [24], PM3 [25], MSINDO [26]). Experimental molecular and lattice structures [3] have been used throughout this study. Note that none of the semiempirical parameters used in the different methods have been optimized to reproduce experimental lattice energies.

\section{Results and Discussion}

\subsection{The experimental lattice energies of $\alpha$ - and $\beta$-oxalic acid $v$ s. the corresponding heats of sublimation}

As mentioned in the introduction, the heats of sublimation $\left(\Delta H_{\text {sub }}\right)$ of the two polymorphic forms of oxalic acid have been determined as 23.4 for the $\alpha$ and $22.3 \mathrm{kcal} / \mathrm{mol}$ for the $\beta$-form $[5,6]$. However, unlike the lattice energy defined above, the heat of sublimation is the change of enthalpy associated with the evaporation of one mole of a crystalline solid resulting in the most stable form of the compound in the gasphase. If there is a significant difference between the total energy of the most stable form of the isolated molecule in the gasphase $\left(E_{\text {tot,gp }}\right)$ and the total energy of a free molecule with a structure as it occurs in the $\operatorname{crystal}\left(E_{\text {tot,cry }}\right)$, then this has to be taken into account by a correction term. In such cases $\Delta H_{\text {sub }}$ and $\Delta E_{\text {lat }}$ are related to each other by the approximate relationship

$$
\Delta E_{\text {lat }}=-\Delta H_{\text {sub }}-2 R T+E_{\text {cry }, \text { gas }},
$$

where

$$
E_{\text {cry }, \text { as }}=E_{\text {tot,gp }}-E_{\text {tot,cry }} .
$$

Determination of the molecular weight of the vapour of anhydrous oxalic acid showed that association of the molecules in the gasphase is negligible [5]. More- 
Table 1. Total $\left(E_{\text {tot }}(\mathrm{i})\right)$ and relative $\left(\Delta E_{1,2}\right)$ energies (in Hartrees and kcal/mol, respectively) of 1 and 2 (cf. Fig. 1) at different levels of $a b$ initio theory. "Full" means that core excitations have been included into the MP2 calculation, while "FC" (frozen core) indicates that they have been omitted.

\begin{tabular}{lccc}
\hline Method & $E_{\text {tot }}(\mathbf{1})$ & $E_{\text {tot }}(\mathbf{2})$ & $\Delta E_{1,2}=E_{\text {tot }}(\mathbf{1})-E_{\text {tot }}(\mathbf{2})$ \\
\hline MP2(Full)/6-31+G*//MP2(Full)/6-31+G* & -377.373710 & -377.370406 & -2.07 \\
MP2(Full)/6-31G**//MP2(Full)/6-31G $G^{* *}$ & -377.369759 & -377.365750 & -2.52 \\
ZPE+MP2(Full)/6-31G**//MP2(Full)/6-31G** & -377.319500 & -377.315959 & -2.22 \\
MP2(FC)/6-311++G**//MP2(FC)/6-311++G** & -377.546754 & -377.542924 & -2.40 \\
ZPE+MP2(FC)/6-311++G**//MP2(FC)6-311++G** & -377.497218 & -377.493943 & -2.06 \\
MP2(FC)/6-31+G*//MP2(FC)/6-31+G* & -377.351377 & -377.348161 & -2.02 \\
MP2(FC)/6-311++G**//MP2(FC)/6-31+G* & -377.545889 & -377.542166 & -2.34 \\
MP2(FC)/6-311++G*//(MP2(FC)/6-311++G**) $\dagger$ & & -377.539875 & -4.32 \\
\hline
\end{tabular}

$\dagger$ Calculated at the geometry of the central molecule in the trimer of oxalic acid.
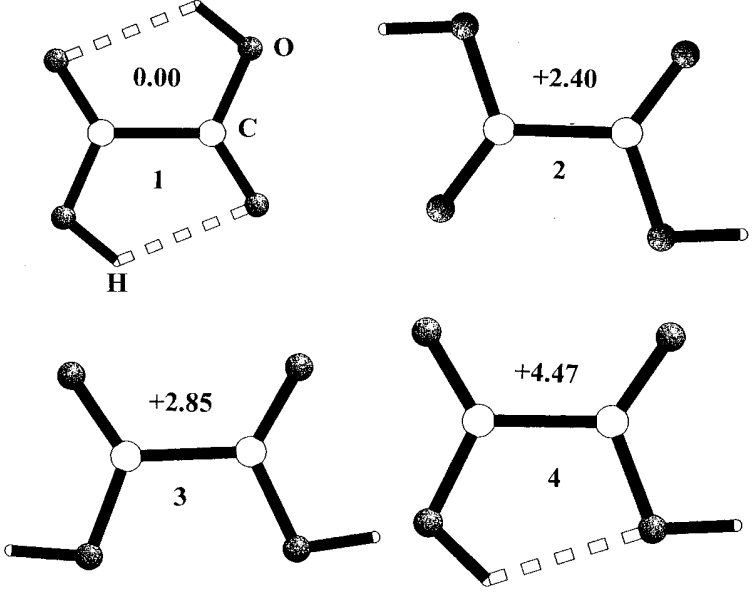

Fig. 1. Some isomers of oxalic acid. The most stable form of oxalic acid in the gasphase and in the solid state are $\mathbf{1}$ and $\mathbf{2}$, respectively. The numbers are energies relative to the most stable isomer 1 obtained at the MP2(FC)/6$311++\mathrm{G}^{* *} / / \mathrm{MP} 2(\mathrm{FC}) / 6-311++\mathrm{G}^{* *}$ level in $\mathrm{kcal} / \mathrm{mol}$.

over, electron diffraction $[27,28]$ and IR studies revealed that free oxalic acid is planar in the gasphase and that the molecule exists in a trans conformation with two intramolecular hydrogen bonds to the carbonyl oxygens of the neighbouring carboxyl groups (cf. Fig. 1, Structure 1). At 1.54(6) [27] and 1.548(4) ^ [28] the bond connecting the two $\mathrm{COOH}$ groups is a single bond, probably slightly elongated relative to the $\mathrm{C}-\mathrm{C}$ bond in ethane ${ }^{\mathrm{d})}$ by repulsion between the electron density accumulated by the oxygen atoms of the carboxyl groups.

To obtain a reliable value for $\Delta E_{\text {cry,gas }}$, quantumchemical calculations have been performed for some

\footnotetext{
d) The $\mathrm{C}-\mathrm{C}$ bond lengths calculated at the MP2(FC)/6-311++G** level are $1.529 \AA$ in ethane and $1.543 \AA$ in $\mathbf{1}$.
}

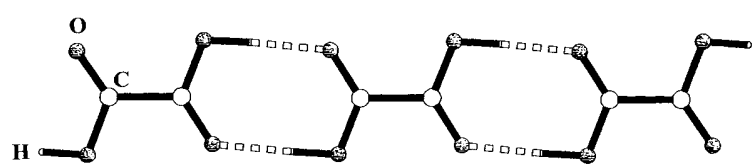

A

B

A

Fig. 2. Oxalic acid trimer optimized at the MP2(FC)/6$311++\mathrm{G}^{* *}$ level under constraint of $C_{2 \mathrm{~h}}$ symmetry.

isomers of oxalic acid at several levels of $a b$ initio theory. The results are shown in Figure 1. The total and relative energies of $\mathbf{1}$ and $\mathbf{2}$ obtained at different levels of precision are given in Table 1. Among the limited number of isomers studied in this paper, only $\mathbf{1}$ is a minimum in the gasphase while $\mathbf{2}$ - $\mathbf{4}$ are saddle points with a single imaginary frequency in the spectra of their normal vibrations. The second stable (extended) isomer, $\mathbf{2}$, is structurally similar to the $(\mathrm{COOH})_{2}$ molecule of both polymorphs in the solid state. At $2.40 \mathrm{kcal} / \mathrm{mol}$ the energy difference between 1 and $\mathbf{2}$ (which has been optimized under constraint of planarity) is relatively small, indicating that the interaction of the protons with the carbonyl oxygen atoms is only slightly more favourable in $\mathbf{1}$ (H…O: $2.109 \AA$ ) than in 2 (H...O: $2.313 \AA$ ). The determination of the true value of $\Delta E_{\text {cry,gas }}$ is hampered by the fact that the structure of the molecule determined in the solid state is not directly comparable to the structures obtained in quantum-chemical calculations. Calculations at the MP2/6-311++G** level using the uncorrected molecular structures as determined by X-ray scattering resulted in energy differences relative to fully optimized 1 of $6.3 \mathrm{kcal} / \mathrm{mol}$ for the $\alpha$ - and $6.0 \mathrm{kcal} / \mathrm{mol}$ for the $\beta$-polymorph. These energy differences are most likely too large, because the structural parameters obtained for the molecules in the solid state are affected by vibrational motions 
Table 2. Lattice energies and their components (in kcal $/ \mathrm{mol}$ ) of $\alpha$ - and $\beta$-oxalic acid, calculated using (scalar) atomic polarizability increments. Experimental molecular geometries have been used in these calculations.

\begin{tabular}{lccccc}
\hline Method & $\Delta E_{\text {dis }}$ & $\Delta E_{\text {els }}$ & $\Delta E_{\text {ind }}$ & $\Delta E_{\text {rep }}$ & $\Delta E_{\text {lat }}$ \\
\hline CNDO/2[22] & & & & & \\
$\alpha$-oxalic acid & -33.07 & -4.22 & -0.81 & +9.94 & -28.16 \\
$\beta$-oxalic acid & -38.34 & -4.92 & -1.08 & +17.26 & -27.08 \\
INDO[23] & & & & & \\
$\alpha$-oxalic acid & -30.75 & -5.84 & -1.07 & +9.65 & -28.01 \\
$\beta$-oxalic acid & -35.56 & -6.77 & -1.44 & +16.81 & -26.96 \\
MINDO/3[19] & & & & & \\
$\alpha$-oxalic acid & -26.31 & -14.17 & -2.44 & +8.57 & -34.35 \\
$\beta$-oxalic acid & -30.35 & -16.16 & -3.26 & +14.88 & -34.89 \\
MNDO[21] & & & & & \\
$\alpha$-oxalic acid & -29.02 & -5.76 & -1.19 & +9.05 & -26.92 \\
$\beta$-oxalic acid & -33.41 & -7.00 & -1.71 & +15.77 & -26.35 \\
AM1[24] & & & & & \\
$\alpha$-oxalic acid & -29.09 & -6.29 & -1.41 & +8.57 & -28.22 \\
$\beta$-oxalic acid & -33.49 & -7.45 & -1.95 & +15.07 & -27.82 \\
PM3[25] & & & & & \\
$\alpha$-oxalic acid & -28.54 & -6.88 & -1.40 & +9.13 & -27.69 \\
$\beta$-oxalic acid & -32.94 & -8.28 & -2.00 & +16.01 & -27.21 \\
MSINDO[26] & & & & & \\
$\alpha$-oxalic acid & -25.90 & -9.63 & -1.89 & +8.05 & -29.37 \\
$\beta$-oxalic acid & -29.73 & -10.76 & -2.32 & +14.22 & -28.59 \\
\hline & & & & &
\end{tabular}

of their atoms resulting in an apparent shortening of the bonds [29, 30]. Moreover, especially the positions of the hydrogen atoms are by no means comparable with those obtained from quantum-chemical geometry optimizations. A better structural model for the molecule in the solid state, resulting in a total energy which is comparable with that calculated for $\mathbf{1}$, is the central molecule $(\mathbf{B})$ in the oxalic acid trimer ${ }^{\mathrm{e})}$ shown in Figure 2. This molecule is certainly more similar to the molecules in the $\beta$ - than in the $\alpha$-modification, but like the molecules in both polymorphs, it is involved in four hydrogen bonds. At the MP2/6-

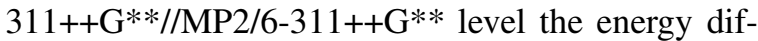
ference between the fully optimized structure $\mathbf{1}$ and this molecule $\mathbf{B}\left(\Delta E_{\text {cry,gas }}\right)$ amounts to $4.3 \mathrm{kcal} / \mathrm{mol}$. Together with the heats of sublimation given in [5] this results in approximate experimental lattice energies of -28.9 and $-27.8 \mathrm{kcal} / \mathrm{mol}$ for the $\alpha$ - and the $\beta$-polymorph, which might be compared with the values calculated with the method described above.

\footnotetext{
e) The total energy of the trimer at the MP2(FC)/6-31++G**// MP2(FC)/6-311++G** level is -1132.676349 a.u. where the geometry has been optimized under constraint of $\mathrm{C}_{2 \mathrm{~h}}$ symmetry. The energy of trimerization is $22.6 \mathrm{kcal} / \mathrm{mol}$ on the same level of theory.
}

\subsection{Semiempirical calculation of the lattice energies}

The lattice energies obtained using scalar atomic polarizabilities [20] are compiled in Table 2. In accordance with the experimental data [5] the results of all calculations obtained in this study agree in that the lattice energies of both modifications are quite similar. Moreover, all but the MINDO/3 method reproduce the experimental finding that the $\alpha$-polymorph is slightly more stable than the $\beta$-form. The shorter hydrogen bonds in the $\beta$-polymorph result in dispersion, induction, and electrostatic energies which are more negative than those calculated for the $\alpha$-form. However, this gain in stabilization energy $\left(\sim r^{-n}\right.$, $n=6,4,1$ ) is overcompensated by the exponentially increasing repulsion energy.

The electrostatic energies calculated in this paper are significantly less stabilizing than those given in [12b] for the $\alpha$-polymorph (average value: $-26.2 \mathrm{kcal} / \mathrm{mol}$ ), and the same is true for the induction energies (average value: $-7.4 \mathrm{kcal} / \mathrm{mol}[12 \mathrm{~b}]$ ). However, together with the corresponding dispersion and repulsion energies in Table 2 they combine to mostly reasonable values for the lattice energies of both polymorphs. Thus, except for the MINDO/3 values, the numerical agreement between the experimental data and the calculated values in Table 2 is surprisingly good if one takes into account that none of the semiempirical parameters had been readjusted to reproduce the experimental lattice energies. Omitting the initially mentioned MINDO/3 data, the average of the absolute values of the differences between the experimental and calculated lattice energies is about $1.0 \mathrm{kcal} / \mathrm{mol}$ for the $\alpha$ - and $0.7 \mathrm{kcal} / \mathrm{mol}$ for the $\beta$ polymorph, where most of the calculated lattice energies are somewhat more positive than the experimental values. Since we are working on a semiempirical level, computational reproduction of the relative stability of the two polymorphs is more important than numerical agreement between observed and calculated values. Averaging over the energy differences obtained from the lattice energies in Table 2 (except the MINDO/3 values) results in a calculated relative stability of about $0.7 \mathrm{kcal} / \mathrm{mol}$ in favour of the $\alpha$ polymorph. This calculated value is quite similar to the experimental result of $1.1 \mathrm{kcal} / \mathrm{mol}$.

\section{Conclusions}

A computational scheme introduced in a preceding paper [18] has successfully been applied to calculate 
the relative stability of the two polymorphs of oxalic acid. The successful treatment of such crystal lattices which are highly crosslinked by hydrogen bonds shows that this method can be used to describe even such strong intermolecular interactions not only without introducing additional potentials but also without altering the current energy expressions.

[1] S. B. Hendricks, Z. Kristallographie 91, 48 (1935).

[2] E. G. Cox, M. W. Dougill, and G. R. Jeffrey, J. Chem. Soc. 1952, 4854.

[3] J. L. Derissen and P. H. Smit, Acta Cryst. B30, 2240 (1974).

[4] Z. Berkovitch-Yellin and L. Leiserowitz, J. Amer. Chem. Soc. 104, 4052 (1982).

[5] R. S. Bradley and S. Cotson, J. Chem. Soc. 1953, 1684.

[6] A. H. Jones, Chem. Eng. Data 5, 196 (1960).

[7] J. L. Derissen and P. H. Smit, Acta Cryst. A34, 842 (1978).

[8] H. G. M. de Wit, J. A. Bouwstra, J. G. Blok, and C. G. de Kruif, J. Chem. Phys. 78, 1470 (1983).

[9] F. A. Momany, L. M. Carruthers, R. F. McGuire, and H. A. Scheraga, J. Phys. Chem. 78, 1595 (1974).

[10] L. G. Dunfield, A. W. Burgess, and H. A. Scheraga, J. Phys. Chem. 82, 2609 (1978).

[11] P. H. Smit, J. L. Derissen, and F. B. Van Duijneveldt, Mol. Phys. 37, 521 (1979).

[12] a) A. Gavezzotti and G. Filippini, J. Phys. Chem. 98, 4831 (1994). b) A. Gavezzotti, J. Phys. Chem. B. 106, 4145 (2002).

[13] S. Lifson, A. T. Hagler, and P. Dauber, J. Amer. Chem. Soc. 101, 5111 (1979).

[14] A. T. Hagler, S. Lifson, and P. Dauber, J. Amer. Chem. Soc. 101, 5122 (1979).

[15] A. T. Hagler, P. Dauber, and S. Lifson, J. Amer. Chem. Soc. 101, 5131 (1979).

[16] I. Nobeli and S. L. Price, J. Phys. Chem. A. 103, 6448 (1999).

[17] T. Beyer and S. L. Price, J. Phys. Chem. B. 104, 2647 (2000).

[18] G. Raabe, Z. Naturforsch. 55a, 609 (2000).

[19] a) R. C. Bingham, M. J. S. Dewar, and D. H. Lo, J. Amer. Chem. Soc. 97, 1285 (1975). b) R. C. Bing-

\section{Acknowledgements}

The author gratefully acknowledges financial support by the Fonds der Chemischen Industrie and helpful discussions with Prof. Dr. Jörg Fleischhauer (Aachen).

ham, M. J. S. Dewar, and D. H. Lo, J. Amer. Chem. Soc. 97, 1294 (1975).

[20] G. Raabe, E. Zobel, R. Kock, and J. P. Souren, Z. Naturforsch. 52a, 665 (1997).

[21] a) M. J. S. Dewar and W. Thiel, J. Amer. Chem. Soc. 99, 4899 (1977). b) M. J. S. Dewar and W. Thiel, J. Amer. Chem. Soc. 99, 4907 (1977).

[22] a) J. A. Pople, D. P. Santry, and G. A. Segal, J. Chem. Phys. 43, S129 (1965). b) J. A. Pople and G. A. Segal, J. Chem. Phys. 43, S136 (1965). c) J. A. Pople and G. A. Segal, J. Chem. Phys.44, 3289 (1966). d) D. P. Santry and G. A. Segal, J. Chem. Phys. 47, 158 (1967).

[23] J. A. Pople, D. L. Beveridge, and P. A. Dobosh, J. Chem. Phys. 47, 2026 (1967).

[24] M. J. S. Dewar, E. G. Zoebisch, E. F. Healy, and J. J. P. Stewart, J. Amer. Chem. Soc. 107, 3902 (1985).

[25] a) J. J. P. Stewart, J. Comput. Chem. 10, 209 (1989). b) J. J. P. Stewart, J. Comput. Chem. 10, 221 (1989).

[26] a) B. Ahlswede and K. Jug, J. Comput. Chem. 20, 563 (1999). b) B. Ahlswede and K. Jug, J. Comput. Chem. 20, 572 (1999).

[27] S. Shibata and M. Kimura, Bull. Chem. Soc. Japan 27, 485 (1954)

[28] Z. Náhlovská, B. Náhlovský, and T. G. Strand, Acta Chem. Scand. 24, 2617 (1970).

[29] C. K. Johnson, Crystallographic Computing, Proc. of the 1969 International Summer School on Crystallographic Computing, Topic F: Analysis of Atomic Thermal Vibrations, p. 220ff, F. R. Ahmed, S. R. Hall, and C. P. Huber (Eds.), Munksgaard, Copenhagen 1969.

[30] C. K. Johnson and H. A. Levy, International Tables for X-ray Crystallography, Vol. IV, p. 312ff, J. A. Ibers and W. C. Hamilton (Eds.) Kluver Academic Publishers, Dordrecht 1989. 